\title{
The trust region method for time-domain full waveform inversion
}

\author{
Peng Lin*, Suping Peng, Yongxu Lu, Wenfeng Du \\ State Key Laboratory of Coal Resources and Safe Mining \\ China University of Mining \& Technology (Beijing) \\ Beijing, China \\ linpeng798@126.com
}

\begin{abstract}
Full waveform inversion (FWI) is an effective inversion method to obtain high resolution and precision seismic imaging. There are a lot of conventional local optimization methods, such as Newton's method and gradient method, to solve the ill-posed nonlinear problem. However, these methods always lead local convergence so that the result of inversion could not be accepted. To confront the problem, we propose a new approach, the trust region method, to solve full waveform inversion problem. Trust region method has strong convergence properties, and the new step is limited to the neighborhood of the current step. To test the capability of this method, we apply it to the model of salt domes and synthetic Marmousi from the timedomain FWI point of view. Inversion results show that the inverted model is very close to the true model and the precision is high. Hence, the trust region method is a promising method for full waveform inversion.
\end{abstract}

Keywords—seismic imaging; full waveform inversion; trust region method; acoustic wave equation;

\section{INTRODUCTION}

With the development of oil and petroleum prospect, the challenge that geologists and geophysicists are confronting is tremendous. Full waveform inversion is a new inversion method, which considers the whole waveform to reconstruct the subsurface parameters. Due to the potential of obtaining the high resolution and precision models, FWI has become a research focus of many scholars in the world. FWI is a leastsquare optimization problem, whose objective function is the misfit between the synthetic and the recorded seismic data. To solve the non-linear and ill-posed problem of FWI, the gradient-based and Newton's methods are the conventional nonlinear optimization methods. Tarantola proposed the timedomain full waveform inversion based on the steepest-descent gradient method for the first time, but the convergence rate of this method was low for the large-scale problem ${ }^{[1]}$. In order to improve the inversion efficiency, Brossier applied the limitedmemory BFGS quasi-Newton method to reconstruct velocity structure based on the elastic wave equation, which made better use of the Hessian matrix information ${ }^{[2]}$. L.Métivier presented a feasible implementation of the truncated Newton (TN) method to the FWI problem, using a second-order adjoint-state approach to calculate Hessian matrix ${ }^{[3-4]}$. TN method increases the inversion robustness, though its computation cost is more expensive than conventional methods.
However, a serious disadvantage of conventional methods is that the gradient-based methods only use the gradient information so that the convergence rate is generally quite low. In addition, the Newton's methods consider the Hessian matrix information into the updated direction iteratively, which could improve the inversion precision. However, from the perspective of computation cost, Hessian matrix is more expensive and requires large memory. It is worth mentioning that these conventional methods may suffer from a wrong result due to the presence of local minima. Due to the strong convergence properties, the trust region method has been applied to the geophysical inversion problem. $\mathrm{Li}$ and Wang combined the trust region method to seismic migration inversion, all of which illustrated this method was effective for ill-posed nonlinear inversion problem ${ }^{[5]}$.

In this paper, we propose the trust region method for the full waveform inversion problem, overcoming the drawbacks of conventional methods ${ }^{[6]}$. This method has strong global convergence properties theoretically, which can produce a better result for full waveform inversion.

\section{THEORY AND METHOD}

Full waveform inversion is an ill-posed and nonlinear problem. The objective function is usually formulated as a least-squares optimization problem. The velocity reconstruction is aimed at minimizing the L2-norm formation of objective function, the unconstrained optimization problem

$$
\min E(\mathbf{m})=\frac{1}{2}\left\|\mathbf{d}_{s \mathrm{yn}}-\mathbf{d}_{c a l}\right\|_{2}^{2}
$$

The mathematical optimization algorithms aim to balance the misfit $E$ between the synthetic $d_{\text {syn }}$ and recorded data $d_{\text {cal }}$. $\|\bullet\|_{2}$ denotes a distance of the Euclidean norm and $\mathrm{m}$ represents velocity model. Generally the conventional line search methods approximate the solution iteratively by a search direction and step along the direction based on the initial assumed model. The model can be updated as

$$
\mathbf{m}_{k+1}=\mathbf{m}_{k}+\alpha_{k} \mathbf{d}_{k}
$$

where $\mathrm{k}$ denotes the current number of iterations, $\alpha$ is the step length obtained by line search methods and $\mathbf{d}$ is the updated direction of model. 
The trust region method has better convergence properties for nonlinear unconstrained problems than line search methods [7]. This method forces the distance between the new and current step length within a certain amount, and avoids being trapped because of large step. The updated amount of parameter is forced within a neighborhood of the current iterate point, which is called the trust region. Compared with the conventional line search algorithms, the trust region method determines the trial step directly according to the model, rather than to determine the search direction firstly, then the step length.

In trust region method, we obtain the updated trial step by solving the trust region subproblem, which is generated from the second-order Taylor expansion of the objective function. The solution is forced within the trust region. The subproblem is defined as

$$
\begin{aligned}
& \min \phi\left(\omega_{k}\right)=\mathbf{g}^{T} \omega_{k}+\frac{1}{2} \omega_{k}^{T} \mathbf{H}_{k} \omega_{k} \\
& \text { s.t. }\left\|\omega_{k}\right\|_{2} \leq \Delta_{k}
\end{aligned}
$$

where $\mathbf{g}$ and $\mathbf{H}$ is the gradient and Hessian matrix of the objective function $^{[8]},\|\cdot\|_{2}$ is the L-2 norm of Euclidean space, and $\mathrm{k}$ denotes the trust region radius.

The solution $\omega_{\mathrm{k}}$ of trust region subproblem is the key problem. Simultaneously, the computation cost of equation (3) is expensive. There are a lot of methods to solve the subproblem, such as the dogleg, double dogleg techniques and the truncated $\mathrm{CG}$ method ${ }^{[9]}$, etc. In this paper, we use the truncated CG method to obtain the solution of equation (3). The parameter $R_{k}$ determines whether the solution of subprolem accepts and how to update the trust region radius, defined as

$$
R_{k}=\frac{A_{k}}{P_{k}}=\frac{E\left(\mathbf{m}_{k}\right)-E\left(\mathbf{m}_{k}+\omega_{k}\right)}{\Phi_{k}(0)-\Phi_{k}\left(\omega_{k}\right)}
$$

where $A_{k}$ denotes the actual reduction of equation(1), and $P_{k}$ is the predicted reduction of equation(3). Generally speaking, if $R_{k}>0$ the solution will be accepted, otherwise, the new step $\omega_{\mathrm{k}}$ is rejected. The trust region radius could be updated as

$$
R_{k}=\frac{A_{k}}{P_{k}}=\frac{E\left(\mathbf{m}_{k}\right)-E\left(\mathbf{m}_{k}+\omega_{k}\right)}{\Phi_{k}(0)-\Phi_{k}\left(\omega_{k}\right)}
$$

where $0<\mathrm{r} 1<\mathrm{r} 2<1,0 \leq \eta 1<\eta 2<1$, all of which are constants. To make it clear, the flow chart is outlined for the trust region method in Fig. 1.

\section{EXAMPLES}

To test the capability of the trust region method, we apply this method to the imaging of salt domes and Marmousi model. The inversion is performed in time-domain acoustic wave equation. The first model of salt domes is inverted shown in
Fig. 2a.The model is divided into $400 \times 150$ grids, and the intervals is $10 \mathrm{~m} \times 10 \mathrm{~m}$. There are 19 shots at intervals of $200 \mathrm{~m}$, and 400 receivers are distributed near the surface every $10 \mathrm{~m}$. The source estimation is Ricker with the dominant frequency $8 \mathrm{~Hz}$. The starting model is generated by smoothing the true model shown in Fig. 2 b.

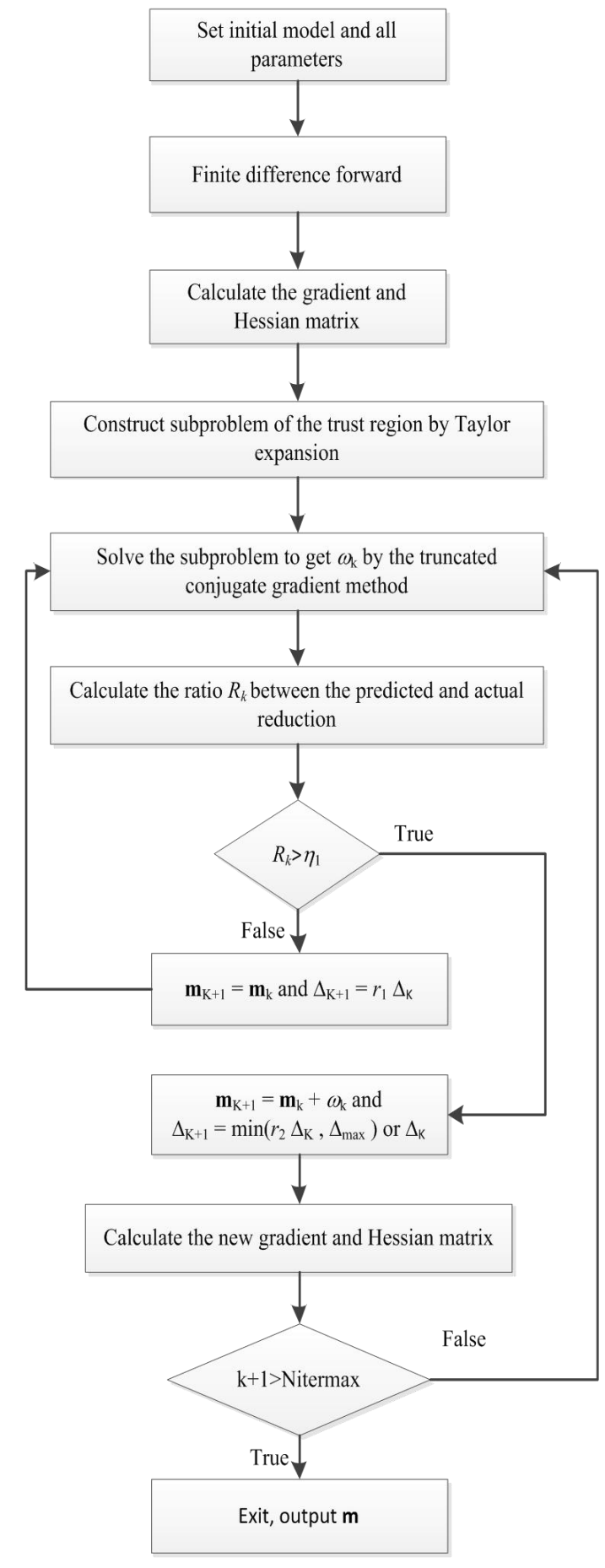

Fig. 1. Flow chart for the trust region method 

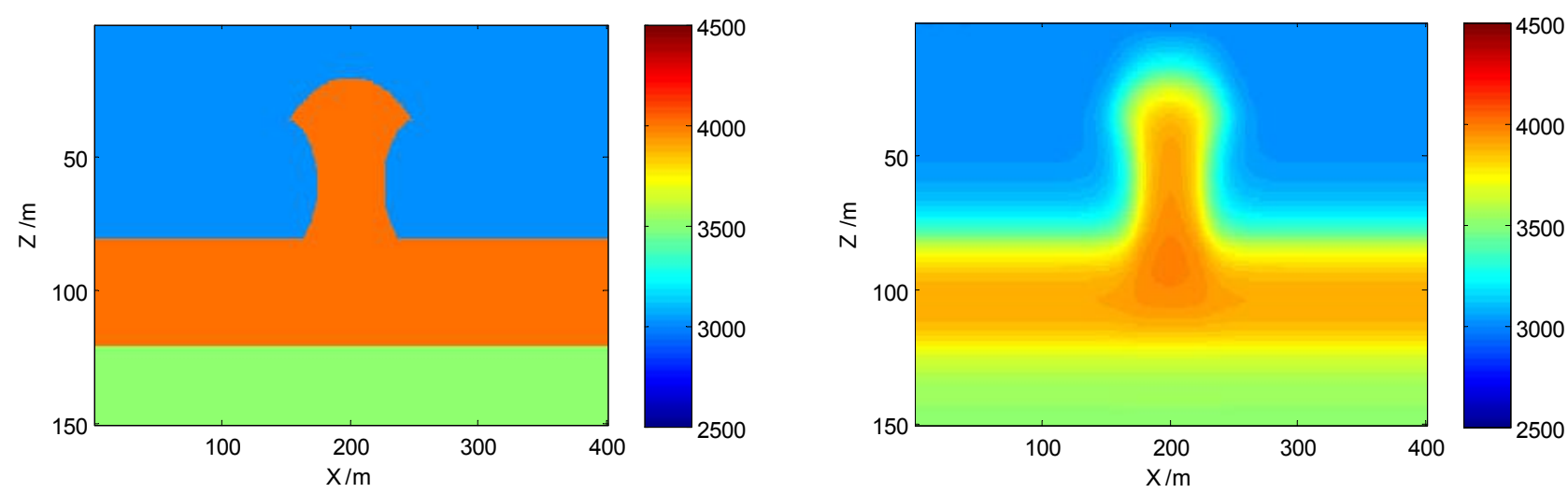

Fig.2. Salt domes model. (a) True model; (b) Starting model obtained from smooth2 function and 20 points in depth, 20 points in crossline direction smoothed by SU
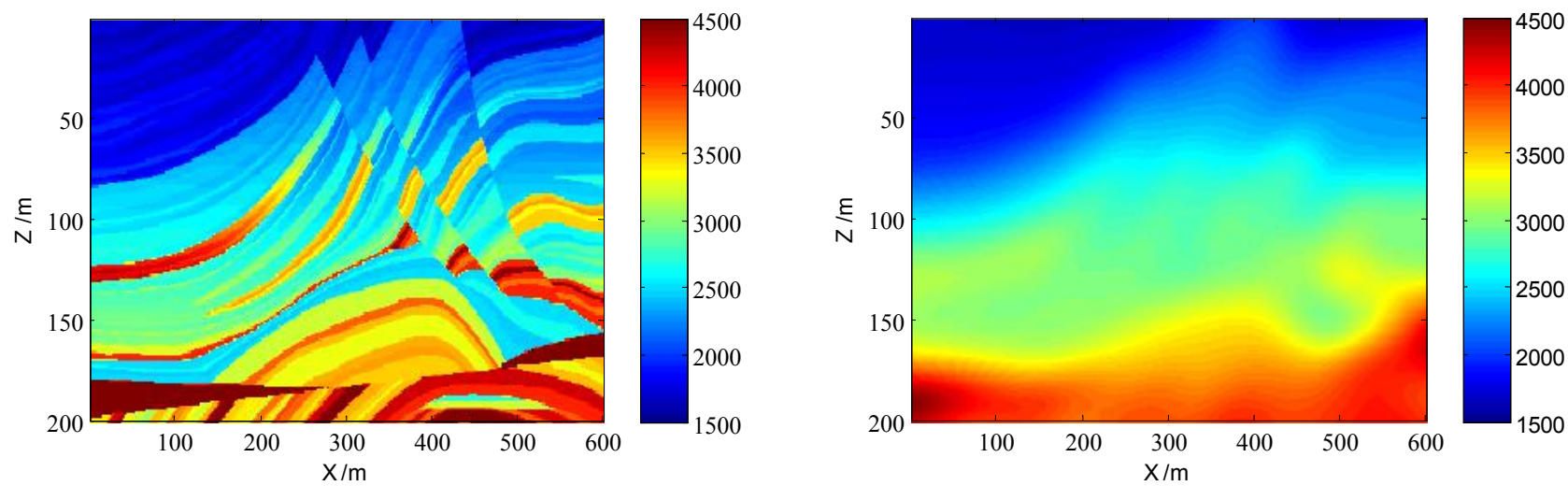

Fig.3. Marmousi model. (a) True model; (b) Starting model obtained from smooth2 function and 10 points in depth, 20 points in crossline direction smoothed by SU
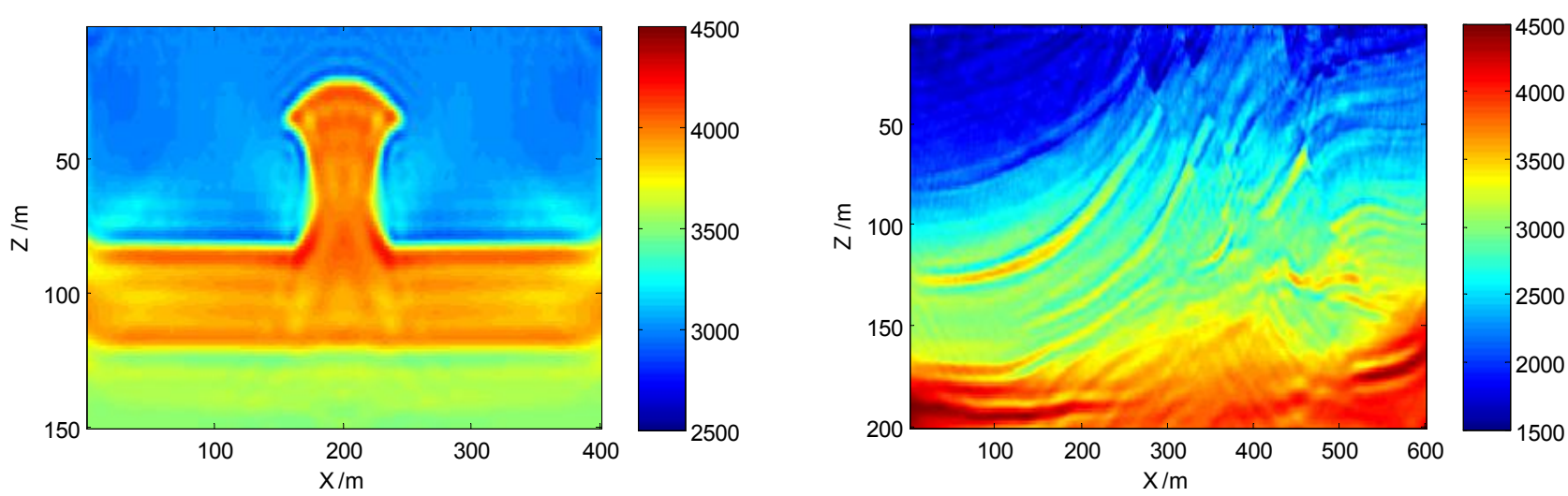

Fig.4. Inversion results. (a) A velocity model of salt domes constructed with the trust region method; (b) A velocity model of Marmousi constructed with the trust region method 
In order to save the computational cost, we cut out a part of Marmousi model as the second model shown in Fig. 3a. The horizontal extent $7200 \mathrm{~m}$ and the depth is $2400 \mathrm{~m}$ in the model which contains $600 \times 200$ grids at intervals of $12 \mathrm{~m} \times 12 \mathrm{~m}$. The acquisition system consists of 19 sources at intervals of $300 \mathrm{~m}$ and 599 receivers every $12 \mathrm{~m}$ along the horizontal surface. We adopt Ricker wavelet to estimate the source, with the dominant frequency $10 \mathrm{~Hz}$. The synthetic data are obtained using the finite difference approach. The true model is smoothed to be used as starting model shown in Fig. 3b. Inversion results are shown in Fig. 4a and Fig. 4b. It is worth to mention that the initial radius is 100 , and the forced maximum radius is 200 . To further illustrate the effectiveness of inversion quantitatively, we display the velocity profiles from the salt domes and Marmousi model respectively shown in Fig. 5.

From figure 2 and 3 , it is obviously that the starting model is far from the true model in detail. Figure 4 shows the inversions result with the trust region method by the model of salt domes and Marmousi. FWI with the trust region method converges fast towards the true model. The structure of salt domes and Marmousi is characterized clearly by the inversion with the trust region method. The inversion result could be improved greatly by the trust region method, especially for the deeper area. In addition, it could be found that the reconstructed velocity model by the trust region method is quite close to the true model, and improve the precision greatly.
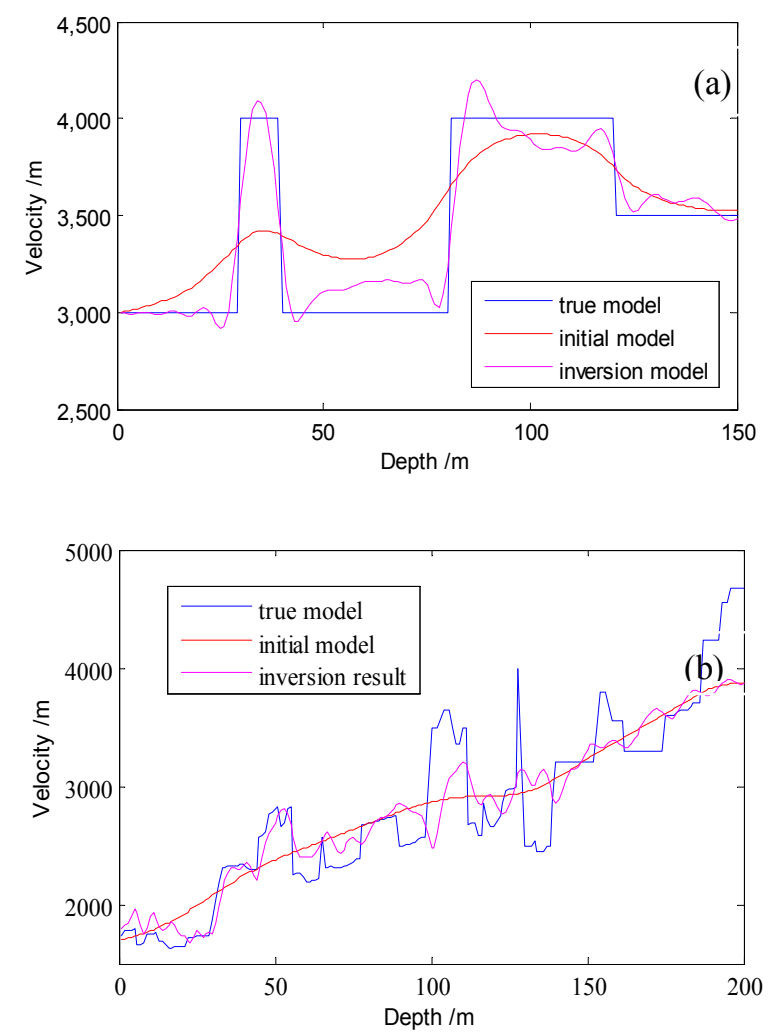

Fig.5. The velocity profiles. (a) The velocity curves of salt domes at distance of $2370 \mathrm{~m}$; (b) The velocity curves of Marmousi model at distance of $2800 \mathrm{~m}$.
The velocity curves show that the inversion result converges to the true model perfectly at the shallow subsurface. But as for the deeper area, the convergence becomes poorer than the shallow subsurface.

\section{CONCLUSIONS}

In the above of this paper, we have demonstrated the trust region method could succeed in full waveform inversion, with the ill-posed and highly nonlinear problem. The trust region method is a robust optimization method with the global convergence different from the conventional line search methods. The conventional line search methods fail to converge to the true model when the problem is large-scale and the starting model is far from the true model, but the trust region method could avoid the presence in theory, improve the inversion accuracy greatly and produce better results, especially for the shallow structure. The trust region method is a promising method for full waveform inversion.

\section{ACKNOWLEDGMENT}

We are grateful for financial supports from National Natural Science Funds Coal Joint Project (U1261203), National Key Scientific Instrument and Equipment Development Program (2012YQ030126), Shanxi Natural Science Funds Project (2013012001).

\section{REFERENCES}

[1] A. Tarantola, "Inversion of seismic reflection data in the acoustic approximation," Geophysics, vol.49, pp. 1259-1266, August 1984.

[2] R. Brossoer, S. Operto, and J. Virieux, "Seismic imaging of complex onshore structures by 2D elastic frequency-domain full-waveform inversion," Geophysics, vol.74, pp. 105-118, Novermber 2009.

[3] L. Metivier, R. Brossier, J. Virieux, and Operto S., "Full waveform inversion and the truncated Newton method," SIAM journal on Scientific Computing, vol.35, pp. 401-437, March 2013.

[4] L. Metivier, Bretaudeau F., Brossier R., Operto S. and Virieux J., "Full waveform inversion and the truncated newton method: quantitative imaging of complex subsurface structures," Geophysical Prospecting, vol.62, pp. 1353-1375, June 2012.

[5] Z. Li and Y. Wang, "A subspace trust-region method for seismic migration inversion," Optimization Methods and Software, vol. 29, pp. 286-296, July 2012.

[6] M. J. D. Powell, "A new algorithm for unconstrained optimization, in: J. B. Rosen, O. L. Manfasarian and K. Ritter, eds," Nonlinear programming, Academic Press, New York, in press.

[7] Y. Wang, Y. Yuan, "Convergence and regularity of trust region methods for nonlinear ill-posed inverse problems," Inverse Problems, vol.21, pp. 821-838, March 2005.

[8] R. E. Plessix, "A review of the adjoint-state method for computing the gradient of a functional with geophysical applications," Geophysical Journal International, vol. 167, pp. 495-503, Novermber 2006.

[9] Y. Yuan, "On the truncated conjugate gradient method," Mathematical Programming, vol. 87, pp. 561-573, May 2000. 